\title{
Wir sind nie revolutionär gewesen - Zum Mythos des Kieler Geographentags als der Geburtsstunde einer neuen Geographie
}

\author{
B. Michel \\ Universität Erlangen-Nürnberg, Institut für Geographie, Wetterkreuz 15, 91058 Erlangen, Germany \\ Correspondence to: B. Michel (boris.michel@fau.de)
}

In der Selbstbeschreibung der deutschsprachigen Geographie westlich des Eisernen Vorhangs trennt der Kieler Geographentag von 1969 zwischen einem Davor und einem Danach, zwischen einer länderkundlichen und einer raumwissenschaftlichen, einer prä-wissenschaftlichen und einer wissenschaftlichen Geographie. Der Kieler Geographentag und der mit diesem assoziierte Durchbruch der „Quantitativen Revolution“ dient den unterschiedlichsten Ansätzen als gemeinsamer Referenzpunkt und bei allen Divergenzen der heute sich als multiparadigmatisch begreifenden Disziplin verbindet sie die Differenz zu einer „Geographie vor Kiel““. Damit wird die Geographie vorher in die Rolle des konstitutiven Außen moderner, wissenschaftlicher und zeitgemäßer Geographien gesetzt. Kiel als epistemologischer Bruch, wissenschaftliche Revolution oder Paradigmenwechsel ist damit ein bedeutsamer und wirkmächtiger Mythos, der die Gegenwart kittet und der deutschsprachigen Geographie sinnhafte Einheit stiftet.

Einführungen in das Studium der Geographie wissen ebenso wie wikipedia und die Geschichtsschreibung der Disziplin, dass das Ende der länderkundlichen Geographie sich „,nicht allmählich“ (Dürr und Zepp, 2012:215) vollzog, sondern hierfür ein markantes Ereignis benannt werden kann. „Mit dem Kieler Geographentag von 1969, auf dem sie als wissenschaftstheoretisch unfundiert, holistisch und naturalistisch kritisiert wurde, verlor die Länderkunde stark an Bedeutung “ (http://de.wikipedia.org/wiki/Länderkunde). „Erst mit der ,quantitativen Revolution“ im Anschluss an den Kieler Geographentag“ (Weichhart, 2008:36), „der berühmten Kieler Revolution“, (ebd, S. 68) wurde Geographie eine moderne und praxisrelevante Wissenschaft. Die alte Geographie, so die Erzählung, erlag einem studentischen Thesenpapier. ,The students' cricism at the Geographical Congress in Kiel was a bombshell for the geography establishment" (Wardenga, 2006:147). Mit der Betonung eines epistemolo- gischen Bruchs, studentischer Rebellion und der Etablierung einer gesellschaftsrelevanten und quantitativen Geographie sind die wesentlichen Momente dieses Narrativs angesprochen.

In diesem kurzen und verkürzt zugespitzten Beitrag möchte ich einige historische Unschärfen betonen und für eine unschärfere Erzählung plädieren. Dabei werden drei Punkte angesprochen: (1) Kiel war kein radikales Ereignis, keine Emergenz, in der Ungehörtes und Unerhörtes geäußert wurde. Vielmehr gibt es eine Vorgeschichte. (2) Kiel veränderte die Geographie nicht. Weder „Revolutionäre“ noch das „Ancien Régime“ sahen das Gesehene als revolutionären Umbruch, sondern eher als eine ausladende und fruchtlose Diskussionsveranstaltung. Es folgte vielmehr eine lange und widersprüchliche Nachgeschichte. (3) Kiel wurde zu „Kiel“ gemacht, die Bedeutung, die „Kiel“ für die deutschsprachige Geographie hat, ist Folge einer aktiven Arbeit am Mythos.

Ziel müsste es daher sein, eine stärker wissenschaftsgeschichtliche (und vermutlich auch wissenschaftssoziologische und wissenschaftsethnologische) Auseinandersetzung zu unternehmen, die sich mit jener Arbeit am Mythos ebenso befasst, wie mit einer Untersuchung der Möglichkeitsbedingungen von „Kiel“. Deutlich würde dabei, dass die Geschichte geographischer Praktiken, die sich um jene Veränderungen gruppieren, die der Begriff „Kiel“ fassen soll, unordentlicher und unreiner waren, als der Mythos.

(1) Die vielfach dem „Bedürfnis nach Anschaulichkeit des geschichtlichen Aktes“ (Blumenberg, 2014:22) geschuldete Fokussierung auf Ereignisse, singuläre Daten oder Brüche verdeckt ermöglichende Strukturen und Machtverhältnisse. Eine wissenschaftliche Revolution, dass wussten vermutlich die während der Diskussionen Thomas Kuhn zitierenden Studierenden in Kiel ebenso wie weite Teile der Wissenschaftsforschung, ist nicht gleich der Durchsetzung des besseren Arguments, sondern ein komplexes soziales Gefüge 
aus widerstreitenden Momenten der Ermöglichung und Verhinderung, der De- und Reterritorialisierung, aus Machtverhältnissen und Pfadlogiken, die dazu führen, dass ein neues Paradigma geglaubt, mit Vertrauen ausgestattet und wirksam werden kann.

Zwar strahlen die Berichte des Geographentags und seiner Diskussionen die ,äußere [...] Gefährdung und innere [...] Unruhe“" (Schöller, 1970:37f) aus, die insbesondere die studentischen Interventionen auslösten. Ein revolutionärer Moment oder ein historisches Ereignis sind diese Diskussionen jedoch höchstens in dem Maße, dass hier etwas bereits präsentes in der Situation der Kopräsenz verstärkt wurde oder das eine Diskussion länger dauerte, als im Tagungsprogramm angekündigt.

Die oben erwähnte „Bombe“ kann kaum überrascht haben. So wie die Geographie im 20. Jahrhundert nie eine Phase erlebte, in der nicht von der einen oder anderen Seite eine Krise der Disziplin proklamiert wurde und von einer Phase als Normalwissenschaft höchstens in retrospektiver Homogenisierung gesprochen werden kann, so war - und darin hatten die Bewahrer der Geographie gegenüber dem studentischen Angriff recht - die Geographie Ende der 1960er Jahre nicht mehr jene der 1920er Jahre. Eine Krise der Länderkunde war im Bewusstsein zahlreicher FachvertreterInnen. Weder in der Wirtschaftsgeographie und Ansätzen der Sozialgeographie, noch in weiten Teilen der physischen Geographie wurde jene Länderkunde betrieben, die Zielscheibe des Angriffs war. Die Erzählung eines Bruchs blendet funktionalistische Ansätze ebenso aus wie die Rezeption von Christaller, zahlreiche Arbeiten, die nicht im länderkundlichen Paradigma aufgehen und nahezu alles, was seit fast 20 Jahren an neuen Ansätzen in der nordamerikanischen, britischen und schwedischen Geographie geschah. Bei allem Solipsismus der deutschsprachigen Geographie, die lange brauchte, ihre neue Randstellung in der internationalen Geographie anzuerkennen, fanden Rezeptionen quantitativ-theoretischer Geographie ebenso statt wie zahlreiche persönliche Kontakte zu einer Geographie jenseits der deutschen Länderkunde, auch wenn sich dies selten in Form veröffentlichter Texte materialisierte.

Das bedeutet nicht, dass es um eine Spurensuche nach frühen Formulierungen einer neuen Raumwissenschaften gehen sollte, etwa danach, ob von Thünen auch schon quantitativ raumwissenschaftliche Geographie betrieben habe oder nicht. Eine solche Fragestellung ist methodologisch so fragwürdig wie sie uninteressant ist. Wichtig ist aber, die wenig revolutionäre Veränderung wissenschaftlicher Diskurse und Praktiken. Selbst ein als so „revolutionär“ begriffener Text wie Bartels Habilitation (Bartels, 1968), war bemüht, Kontinuität zu behaupten und Anschluss herzustellen. Einer Wissenschaftsgeschichte von „Kiel“ müsste es darum gehen, zu klären, was nötig wurde, damit das in Kiel geäußerte zu einem Kristallisationskern werden konnte, der sich seit den 1970er Jahren anbot, einen Mythos zu begründen.
(2) Als zweiter Punkt ist zu betonen, dass nach Kiel möglicherweise nicht so viel anders, bzw. dass die Veränderungen weder unmittelbar noch eindeutig waren. Zwar wird in den Berichten des Geographentags deutlich, dass 1969 als außergewöhnlicher Geographentag wahrgenommen und die dort formulierte Krise in anderen Zusammenhängen weiterdiskutiert wurden. Aber bereits die Berichte zum folgenden Geographentag lassen jeden Gestus von Aufbruch vermissen. Zwar hält auf dem Erlanger Geographentag 1971 Hütteroth einen Vortrag zur ,Wideranknüpfung an die Kieler Diskussion “ (Hütteroth, 1972), aber gerade darin wird deutlich, dass der Kieler Aufbruch eingehegt wurde, dass die Fragen nach Methodologie und Wissenschaftstheorie, nach Gesellschaftsrelevanz oder Gesellschaftskritik auf Fragen der angewandten Planung und Reformulierung des Curriculums reduziert wurden (siehe auch: Belina, 2009). So hebt Hütteroth hervor, dass es nicht die fachlichen Auseinandersetzungen um Länderkunde waren, die relevant blieben, sondern die verwaltungstechnisch formulierte Frage des Praxisbezugs zur „optimalen Bedürfnisbefriedigung“ in einer „Massengesellschaft" (Hütteroth, 1972:170). Zwar warnt Schöller in seiner abschließenden Ansprache in Kiel davor, die aufgebrochenen Fragen beiseite zu wischen: „So würde ich es für falsch und gefährlich halten, wenn einige von uns diesen Geographentag mit dem Gedanken verließen: Wir sind noch einmal davongekommen. Das wäre gefährlich, weil es nicht stimmt" (Schöller, 1970:38). Er hegt dies im folgenden Abschnitt aber dahin gehend ein, dass der Konflikt und die Verletzung der „geographischen Tischsitten“, wie es im Geografiker von 1970 heißt, von einem inhaltlichen Konflikt zu einem Problem des Coming of Age erklärt und eben jede Generation von neuem ,[d]ie Einheit des Faches, die Wissenschaftlichkeit der Länderkunde, die Praxisnähe von Forschung und Ausbildung, die gesellschaftliche und staatspolitische Relevanz der Geographie“ zu entdecken habe (Schöller, 1970:38).

Gegenüber einer durchaus radikalen quantitativtheoretischen Wende in der englischsprachigen Geographie der 1950er und 1960er Jahre, setzt sich in der deutschsprachigen Geographie zudem eine eher halbherzige Distanzierung von der Länderkunde durch. Es trifft zu, wenn Wirth in seinem Nachruf auf Bartels (1984) schreibt, dass in den 1970er Jahren eben nicht eine Humangeographie dominant wird, wie sie Bartels forderte. Und so sind die Einschätzungen von sowohl wissenschaftstheoretisch wie politisch radikaleren VertreterInnen in den frühen 1970er Jahren ziemlich skeptisch (Bahrenberg, 1973:142; Schmidt, 1971; Böttcher, 1970). Die 1970er Jahre waren voller Konflikte, der Neuformulierung und Verteidigung länderkundlicher Ansätze wie auch der Gegenentwürfe und der „konservativen Umarmung“ der Revolution (Bartels, 1980). Länderkunde wurde - oft in geänderter Terminologie - weiterbetrieben und die 1970er Jahre waren eine Zeit zahlreicher „konterrevolutionärer“ Gegenentwürfe (z.B. Schmithüsen, 1976). Möglicherweise, das deutet Barnes 
an, bezog die Quantitative Revolution ihre Stärke zunächst mehr aus der Erfindung einer neuen Sprache und neuen Metaphern, denn aus einer tiefgreifenden Veränderung disziplinärer Gewissheiten (Barnes, 1996).

Statt von einem radikalen Bruch zu sprechen, wäre zu klären, was ausgelöst wurde. Was waren die Momente der Verschiebung, welche Räume wurden geöffnet und welche wie und wann geschlossen, welche Bewegungen der Institutionalisierung und akademischer Karrieren (und deren Verhinderung) führten zu Verschiebungen der Kräfteverhältnisse (oder auch nicht). Es wäre vermutlich produktiver von einem längerfristigen ,Stellungskrieg“ (Gramsci) um disziplinäre Hegemonie zu sprechen, statt einen radikalen Bruch anzunehmen, der das Resultat einiger weniger textlicher oder mündlicher Äußerungsakte war.

Zweifelsfrei fanden in den späten 1960er und frühen 1970er Jahren Veränderungen in der Geographie statt, wurde eine kritische Auseinandersetzung mit der Disziplin in neuer Weise möglich, wurden internationale und zunehmend kritische Beiträge stärker rezipiert und fand eine Hinwendung zu aktuellen gesellschaftlichen Problemen statt. Lediglich das Datum „Kiel 1969“ scheint unpassend, dies zu markieren. Wenn Troll Ende des Jahres von einem ,epochalen Ereignis des abgelaufenen Jahres 1969“ schreibt, so meinte er damit eben nicht den Kieler Geographentag, sondern sprach von der Landung der ersten Menschen auf dem Mond (Troll, 1969).

(3) Das interessanteste an Mythen, Traditionen und großen Erzählungen ist weniger die Frage, ob sie wahr sind, sondern wie sie gemacht wurden und was ihre produktiven Momente bei der Erklärung und Legitimierung der Gegenwart sind. Daher reicht es nicht, die Geschichte von „Kiel“ durch heterogenere Erzählungen, durch Gegenerzählungen oder dekonstruktivistische Lektüren zu ergänzen. Ein Mythos wird zu einem solchen dadurch, dass daran gearbeitet wird. Dieses Arbeiten gälte es nachzuzeichnen und hat vermutlich wenig damit zu tun, was 1969 in einer Sitzung auf einem Geographentag geschah. Daher stellt sich die Frage, wann und wie Kiel zu „Kiel“ wurde und welche Funktion dieses „Kiel“ für die heutige Geographie hat. Der durch dieses Ereignis eingezogene Bruch entlastet die gegenwärtige Geographie schließlich nicht nur in dem Sinne, dass er die Geschichte vereinheitlicht und nützliche Begriffe und Kategorien für die Gegenwart bereithält, sondern auch dadurch, dass er von einer Traditionslinie entlastet, die vermutlich nicht so unsichtbar und unsagbar geworden ist, wie es scheint. Dagegen und das ist ein Plädoyer für eine intensivere Arbeit an der Geschichte der Disziplin - gälte es beispielsweise die Kontinuitäten und Reaktivierungen länderkundlicher Motive zu reflektieren, wie diese möglicherweise in einer vielfach um Unmittelbarkeit und Ganzheitlichkeitsversprechen bemühten Anwendung des Assemblage-Begriffs oder in non represenational theories in der Geographie aufscheint.

\section{Literatur}

Bahrenberg, G.: Zur wissenschaftstheoretischen Grundlegung einer Geographie des Menschen, Geogr. Z., 61, 141-142, 1973.

Barnes, T.: Logics of Dislocation. Models, Metaphors, and Meanings of Economic Space, New York, Guilford, 1996.

Bartels, D.: Zur wissenschaftstheoretischen Grundlegung einer Geographie des Menschen, Wiesbaden, Franz Steiner, 1968.

Bartels, D.: Die konservative Umarmung der "Revolution". Zu Eugen Wirths Versuch in Theoretischer Geographie, Geogr. Z., 68, 121-131, 1980.

Belina, B.: Theorie, Kritik und Relevanz in der deutschsprachigen sozialwissenschaftlichen Geographie 40 Jahre nach Kiel, mit einigen bescheidenen Vorschlägen letztgenannte im Arbeitsalltag als gesellschaftliche zu füllen, Rundbrief Geographie, 221, 1820, 2009.

Blumenberg, H.: Präfiguration. Arbeit am politischen Mythos, Berlin, Suhrkamp, 2014.

Böttcher, H.: 37. Deutscher Geographentag, Geografiker, 4, 3-9, 1970.

Dürr, H. and Zepp, H.: Geographie verstehen. Ein Lotsen- und Arbeitsbuch, Paderborn, Schöningh, 2012.

Hütteroth, W.-D.: Wiederanknüpfung an die Kieler Diskussion, in: Deutscher Geographentag Erlangen-Nürnberg 1. bis 4. Juni 1971. Tagungsbericht und wissenschaftliche Abhandlungen, Herausgeber: Schöller, P. und Liedtke, H., Wiesbaden: Franz Steiner, 169-173, 1972.

Schmidt, E.: Die Selbstdarstellung der Deutschen Geographie auf dem 38. Deutschen Geographentag in Erlangen/Nürnberg, Pfingsten 1971, Roter Globus, 2, 1-8, 1971.

Schmithüsen, J.: Allgemeine Geosynergetik. Grundlagen der Landschaftskunde, Berlin, de Gruyter, 1976.

Schöller, P.: Ansprache des neuen 1. Vorsitzenden des Zentralverbandes Deutscher Geographen, in: Verhandlungen des deutschen Geographentages 37, Herausgeber: Meckelein, W. und Borchererdt, C., Wiesbaden, Franz Steiner, 37-38, 1970.

Troll, C.: Selenographie und Geographie: Ein Rückblick auf das Jahr 1969, Erdkunde, 23, 326-328, 1969.

Wardenga, U.: German geographical thought and the development of Länderkunde, Inforgeo, 18-19, 127-147, 2006.

Weichhart, P.: Entwicklungslinien der Sozialgeographie. Von Hans Bobek bis Benno Werlen, Stuttgart, Steiner, 2008.

Wirth, E.: Dietrich Bartels 1931-1983, Geogr. Z., 72, 1-22, 1984. 